\title{
Piperlongumine inhibits cancer stem cell properties and regulates multiple malignant phenotypes in oral cancer
}

\author{
YIN-JU CHEN ${ }^{1-5}$, CHIA-CHUN KUO ${ }^{1}$, LAI-LEI TING ${ }^{1}$, LONG-SHENG LU ${ }^{1-3}$, YA-CHING LU ${ }^{6}$, ANN-JOY CHENG ${ }^{6}$,

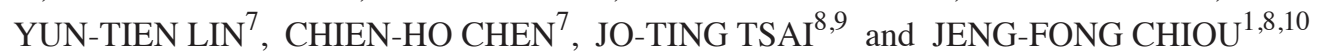 \\ ${ }^{1}$ Department of Radiation Oncology, Taipei Medical University Hospital; ${ }^{2}$ Graduate Institute of Biomedical Materials and \\ Tissue Engineering; ${ }^{3}$ International Ph.D. Program in Biomedical Engineering, College of Biomedical Engineering, \\ Taipei Medical University; ${ }^{4}$ Translational Laboratory, Research Department, Taipei Medical University Hospital; \\ ${ }^{5}$ School of Biomedical Engineering, College of Biomedical Engineering, Taipei Medical University, Taipei 11031; \\ ${ }^{6}$ Department of Medical Biotechnology, Medical College, Chang Gung University, Taoyuan 33302; ${ }^{7}$ School of \\ Medical Laboratory Science and Biotechnology, College of Medical Science and Technology, Taipei Medical University; \\ ${ }^{8}$ Department of Radiology, School of Medicine, College of Medicine, Taipei Medical University, Taipei 11031; \\ ${ }^{9}$ Department of Radiation Oncology, Shuang Ho Hospital, Taipei Medical University, New Taipei City 23561; \\ ${ }^{10}$ Taipei Cancer Center, Taipei Medical University, Taipei 11031, Taiwan, R.O.C.
}

Received July 4, 2017; Accepted September 13, 2017

DOI: $10.3892 / \mathrm{ol} .2017 .7486$

\begin{abstract}
Piperlongumine (PL), a natural product of Piper longum, inhibits multiple malignant phenotypes. Therefore, the present study examined whether PL suppresses cancer stemness in oral cancer. The cellular effects of PL were determined by examining alterations in tumor sphere formation, cell migration, invasion, proliferation ability, chemosensitivity and radiosensitivity. Reverse transcription-quantitative polymerase chain reaction analysis and western blotting were performed in order to determine molecular expression levels. The present study revealed that PL inhibited cancer stem cell-forming ability and suppressed the expression of the stemness-related transcription factors SRY-Box 2, POU class 5 homeobox 1, and Nanog homeobox. However, it increased the expression of the differentiation marker cytokeratin 18. PL also suppressed cell migration and invasion, resulting in the elimination of the epithelial-mesenchymal transition. Furthermore, PL increased chemo- and radiosensitivity and suppressed tumor growth in vitro and in vivo. The results of the present
\end{abstract}

Correspondence to: Dr Jo-Ting Tsai, Department of Radiation Oncology, Shuang Ho Hospital, Taipei Medical University, 291 Zhongzheng Road, Zhonghe, New Taipei City 23561, Taiwan, R.O.C.

E-mail: kitty4024@gmail.com

Dr Jeng-Fong Chiou, Department of Radiation Oncology, Taipei Medical University Hospital, 252 Wu-Xing Street, Taipei 11031, Taiwan, R.O.C.

E-mail: solomanc@tmu.edu.tw

Key words: cancer stem cells, epithelial-mesenchymal transition, piperlongumine, chemosensitivity, radiosensitivity, oral cancer study suggested that PL inhibits malignant phenotypes via the suppression of cancer stemness in oral cancer. Thus, PL may serve as an effective therapeutic agent for oral cancer.

\section{Introduction}

Cancer stem cells (CSCs), or tumor-initiating cells, are a rare population of tumor cells with stem cell-like characteristics and self-renewing abilities that are responsible for cancer maintenance $(1,2)$. CSCs are tumorigenic and metastatic, and are more resistant to chemotherapy and radiotherapy compared with differentiated tumor cells $(3,4)$. Furthermore, CSCs persist in tumors, resulting in relapse following chemotherapy. Thus, developing novel target drugs in order to diminish CSC resistance to chemotherapy or radiotherapy may result in improving therapeutic efficacy and patient prognosis (4).

Most cancer-associated mortalities are caused by metastasis (5), and numerous processes and molecules are involved in this malignant phenotype. Cancer cells disseminate from the primary site to another organ site via the blood, the lymphatic system or seeding. When metastasis is initiated, epithelial cells lose their cell-cell adhesion capacity and cell polarity, and gain migratory and invasive properties, forming mesenchymal cells, a process termed the epithelial-mesenchymal transition (EMT) (6). EMT is characterized by the downregulation of epithelial markers including epithelial (E)-cadherin, cytokeratin (CK)-8, CK-18, CK-19, claudins, occludins and the upregulation of mesenchymal markers including neural (N)-cadherin, fibronectin, vimentin and tenascin C (6). EMT is also regulated by transcription factors including Twist family BHLH transcription factor 1, Snail family transcriptional repressor (Snail)1, Snail2 (also known as Slug), Zinc finger E-Box binding homeobox (ZEB)1 and ZEB2 (7). EMT is a crucial process which contributes to the development of drug resistance and cancer stemness, and the expression level 
of EMT-associated markers predicts patient prognosis and therapeutic effectiveness (8-10).

Oral cancer is one of the most frequently occurring cancers globally, and is particularly prevalent in Southeast Asia (11). Surgery, radiotherapy, chemotherapy or combinations of these methods are the main therapeutic methods used for oral cancer (12). However, the 5-year survival rates of patients with stage I, II, III and IV oral cavity cancer are $72-90 \%$, $39-85 \%, 27-70 \%$ and $12-50 \%$, respectively (13-15). The overall prognosis of advanced-stage oral cancer has not substantially improved in the past two decades $(13,15)$. Therefore, identifying a novel therapeutic method is imperative.

Piperlongumine (PL), a natural product of the plant Piper longum, is a potent anti-inflammatory, antiatherosclerotic and antitumor agent. PL suppresses the production of tumor necrosis factor- $\alpha$ and interleukin- 6 , and inhibits the activation of nuclear factor- $\kappa \mathrm{B}(\mathrm{NF}-\kappa \mathrm{B})$ against proinflammatory responses $(16,17)$. Furthermore, PL prevents plaque formation and inhibits vascular smooth muscle cell migration and invasion via the inhibition of $\mathrm{NF}-\kappa \mathrm{B}$ activation in atherosclerotic lesions (18). The selective tumoricidal effect of PL against cancer cells, but not normal cells, has been demonstrated in various types of cancer including breast cancer, hepatocellular carcinoma, colon cancer, leukemia, glioblastoma, head and neck cancer and prostate cancer (19-22). PL induces the accumulation of reactive oxygen species (ROS), resulting in the apoptosis of cancer cells (19,23-25). This occurs through various mechanisms including the activation of caspases, the inhibition of cyclin dependent kinase (CDK)1, CDK2 and cyclin D1, and the downregulation of antiapoptotic genes including B-cell lymphoma 2 (BCL2), Raf-1 proto-oncogene, serine/threonine kinase and surviving $(25,26)$. In addition, $\mathrm{PL}$ inactivates the phosphatidylinositol-3-kinase/protein kinase $\mathrm{B}$ (Akt)/mammalian target of rapamycin, mitogen-activated protein kinase $14 / \mathrm{c}-J u n$ N-terminal kinase, $\mathrm{NF}-\kappa \mathrm{B}$, and signal transducer and activator of transcription 3 (STAT3) pathways $(20,21,25)$. These pathways serve functions in the regulation of self-renewal and differentiation of CSCs (27-29).

The present study examined whether PL regulates the properties of CSCs which are associated with tumor malignancy and the therapeutic resistance of cancer cells. It was demonstrated that PL suppressed CSC-forming ability and the expression of the CSC-associated markers SRY-box 2 (SOX2), POU class 5 homeobox 1 (Oct-4), and Nanog homeobox (NANOG). PL inhibited cell migration and invasion, and regulated EMT, in addition to increasing chemo- and radiosensitivity and inhibiting tumor growth in vitro and in vivo. These results suggest that PL inhibits numerous malignant phenotypes via the suppression of cancer stemness in oral cancer.

\section{Materials and methods}

Cell culture. The oral cancer cell lines SAS and CGHNC8 (30) were used. SAS cells were kindly provided by Professor S.C. Lin, Yang Ming University (Taipei, Taiwan) and CGHNC8 cells were derived from oral cancer squamous cell carcinomas of patients from Chang Gung Memorial Hospital (Taoyuan, Taiwan). These cells were cultured in Dulbecco's modified Eagle's medium (DMEM; Gibco; Thermo Fisher Scientific, Inc., Waltham, MA, USA) supplemented with $10 \%$ fetal bovine serum (FBS; Biological Industries, Cromwell, CT, USA) and antibiotics (100 U/ml penicillin, $100 \mathrm{U} / \mathrm{ml}$ streptomycin and $0.25 \mathrm{~g} / \mathrm{ml}$ amphotericin B) (Invitrogen; Thermo Fisher Scientific, Inc.) at $37^{\circ} \mathrm{C}$ in $5 \% \mathrm{CO}_{2}$.

Chemicals and antibodies. PL (Sigma-Aldrich; Merck KGaA, Darmstadt, Germany) was dissolved in $100 \%$ dimethyl sulfoxide stock solution and subsequently diluted using the culture medium. The following primary antibodies were used in the present study: Anti-E-cadherin (cat. no. 24E10, dilution, 1:1,000), anti-N-cadherin (cat. no. D4R1HN, dilution, 1:1,000), anti-vimentin (cat. no. D21H3, dilution, 1:1,000; Cell Signaling Technology, Inc., Danvers, MA, USA), anti-Snail (cat. no. SC-28199, dilution, 1:1,000), anti-Slug (cat. no. SC-10436, dilution, 1:1,000), anti-Oct-4 (cat. no. SC-9081, dilution, 1:1,000), and anti-CK18 (cat. no. SC-6259, dilution, 1:1,000; Santa Cruz Biotechnology, Inc., Dallas, TX, USA), anti-NANOG (cat. no. ab109250, dilution, 1:1,000; Abcam, Cambridge, UK), anti-SOX2 (cat. no. AB5603, dilution, 1:1,000; EMD Millipore, Billerica, MA, USA), and anti-GAPDH (cat. no. GTX100118, dilution, 1:8,000; GeneTex, Inc., Irvine, CA, USA).

Tumor sphere formation. For tumor sphere formation, cells were cultured in DMEM/nutrient mixture F-12 (F12) serum-free medium (Gibco; Thermo Fisher Scientific, Inc.) containing $10 \mathrm{ng} / \mathrm{ml}$ epidermal growth factor, $10 \mathrm{ng} / \mathrm{ml}$ human recombinant basic fibroblast growth factor, and B27 supplement (Invitrogen; Thermo Fisher Scientific, Inc.). A diluted cell suspension $(3,000$ cells $/ \mathrm{ml})$ was seeded in ultralow attachment plates (Corning Incorporated, Corning, NY, USA) then incubated at $37^{\circ} \mathrm{C}$ for 2 weeks and fresh medium was added to each well every 4 days. After 2 weeks, the tumor spheres were visualized and enumerated through a Nikon ECLIPSE Ti inverted light microscope.

Cell migration and invasion assays. Cell migration ability was evaluated using an in vitro wound healing assay. Cells were seeded in ibidi ${ }^{\circledR}$ culture inserts (Applied BioPhysics, Inc., Troy, NY, USA) on 6-well plates. Following incubation at $37^{\circ} \mathrm{C}$ for $8 \mathrm{~h}$, the culture insert was detached, forming a $500 \mu \mathrm{m}$ cell-free gap in the cell monolayer. Subsequently, the medium was replaced with new medium containing $1 \%$ FBS and 2.5 and $5 \mu \mathrm{M}$ PL, and cell migration towards the gap area was photographed every $5 \mathrm{~h}$ by Nikon ECLIPSE Ti inverted light microscope. The cell invasion assay was performed using BD BioCoat Matrigel invasion chambers (BD Biosciences, Franklin Lakes, NJ, USA) and Millicell invasion chambers (EMD Millipore). The membrane of the Millicell upper chamber insert (pore size, $8 \mathrm{~mm}$ ) was placed in a $24-$ well plate and coated with Matrigel. Cells in DMEM with 1\% FBS were seeded in the upper chambers. The lower chambers contained DMEM with $10 \%$ FBS in order to trap the invading cells. Cells $\left(1 \times 10^{5}\right)$ were seeded in the upper chambers, with 2.5 and $5 \mu \mathrm{M}$ concentrations of PL for $24 \mathrm{~h}$ and incubated at $37^{\circ} \mathrm{C}$. Cell invasion ability was determined by staining the cells with crystal violet for $30 \mathrm{~min}$ at room temperature that successfully passed through the Matrigel-coated membrane to the opposite chamber and invaded cells was photographed by Nikon ECLIPSE Ti light microscope. 
Radio- and chemosensitivity assays. To examine whether PL serves as a radiosensitizer, a clonogenic assay was conducted. A total of $10^{3}$ cells were seeded in 6-well dishes and incubated for $16 \mathrm{~h}$. Following treatment with $0.625 \mu \mathrm{M}$ PL for $24 \mathrm{~h}$ at $37^{\circ} \mathrm{C}$, the cells were exposed to $2 \mathrm{~Gy}$ of radiation. Subsequently, the medium was replaced with DMEM with $10 \%$ FBS. The cells were incubated at $37^{\circ} \mathrm{C}$ for 5-7 days and stained using crystal violet for $30 \mathrm{~min}$ at room temperature. The results of the radiosensitivity assay were similar to those of the clonogenic survival assay. The number of surviving colonies (defined as a colony with $\geq 50$ cells) were counted The survival percentage was calculated using the following equation: Survival percentage $=$ (number of colonies of treated cells)/(number of colonies of control cells) x100. The colonies were counted using ImageJ software (version 2.0; National Institutes of Health, Bethesda, MD, USA). To determine chemosensitivity, a total of 3,000 cells/well were seeded in 96-well plates. After $16 \mathrm{~h}$, the medium was removed, and $0.03 \mu \mathrm{M}$ 5-fluorouracil (5-FU) (Sigma-Aldrich; Merck KGaA) was added alone or in combination with $2.5 \mu \mathrm{M}$ PL in the DMEM medium. After $48 \mathrm{~h}$ incubated at $37^{\circ} \mathrm{C}$, cell viability was determined using the MTS assay (Promega Corporation, Madison, WI, USA) according to the manufacturer's protocol and measured the absorbance at $490 \mathrm{~nm}$. The relative cell viability was calculated using the following equation: Cell viability $=($ absorbance of treated cells $) /($ absorbance of control cells) x100.

Cell growth and colony formation assays. Cells were seeded at a density of $5 \times 10^{4}$ in 6 -well plates for $16 \mathrm{~h}$ and subsequently treated with $5 \mu \mathrm{M}$ PL for $24 \mathrm{~h}$ and $48 \mathrm{~h}$ at $37^{\circ} \mathrm{C}$. The extent of cell growth was determined daily using a hemocytometer. For colony formation analysis, $10^{3}$ cells were seeded in 6-well plates for $16 \mathrm{~h}$, treated with 0.625 and $2.5 \mu \mathrm{M}$ PL, and allowed to grow uninterrupted for 7 days at $37^{\circ} \mathrm{C}$ in DMEM containing $20 \%$ FBS. Cell colonies were counted following staining with $5 \%$ crystal violet for $15 \mathrm{~min}$ at room temperature.

$R N A$ extraction and reverse transcription-quantitative polymerase chain reaction $(R T-q P C R)$. Total RNA was extracted using the Quick-RNA MiniPrep kit (Zymo Research Corp., Irvine, CA, USA) according to the manufacturer's protocol. The concentration, purity, and amount of total RNA were quantified using the Nano-Drop ND-1000 ultraviolet spectrophotometer (NanoDrop Technologies; Thermo Fisher Scientific, Inc.). Reverse transcription was conducted as previously described (31). qPCR was performed using the Fast SYBR ${ }^{\circledR}$-Green Master Mix (Invitrogen; Thermo Fisher Scientific, Inc.) on the ABI Step One Real-Time PCR system (ABI Corporation, Lee's Summit, MO, USA), according to the manufacturer's protocol. The thermal cycling conditions were an initial denaturation step at $95^{\circ} \mathrm{C}$ for $10 \mathrm{~min}, 40$ cycles at $95^{\circ} \mathrm{C}$ for $15 \mathrm{sec}$ and $60^{\circ} \mathrm{C}$ for $1 \mathrm{~min}$. A comparison RT-PCR method was used to assay the relative expression of the genes in the control and PL treated groups, the expression level of each gene was determined using the $\Delta \Delta \mathrm{Cq}$ method as described previously (32). The following oligonucleotide sequences were used: SOX2 forward, 5'-GCCGAGTGGAAACTTTTGTCG-3' and reverse, 5'-GGCAGCGTGTACTTATCCTTCT-3'; NANOG forward, 5'-TTTGTGGGCCTGAAGAAAACT-3' and reverse,
5'-AGGGCTGTCCTGAATAAGCAG-3'; Oct-4 forward, 5'-CTTGAATCCCGAATGGAAAGGG-3' and reverse, 5'-GTGTATATCCCAGGGTGATCCTC-3'; and GAPDH forward, 5'-CGGAGTCAACGGATTTGGTCGTATG-3' and reverse, 5'-AGCCTTCTCCATGGTGGTGAAGA-3'. GAPDH expression was used as a reference.

Western blotting. Protein extraction and western blotting were performed as previously described (33). Cells were collected and lysed in CHAPS lysis buffer (10 mM Tris, pH 7.4, $1 \mathrm{mM}$ $\mathrm{MgCl}_{2}, 1 \mathrm{mM}$ EGTA, $150 \mathrm{mM} \mathrm{NaCl}, 0.5 \%$ CHAPS and $10 \%$ glycerol; Sigma-Aldrich; Merck KGaA) containing a protease inhibitor. For protein separation, $30 \mu \mathrm{g}$ of protein was electrophoresed on $10 \%$ sodium dodecyl sulfate-polyacrylamide gel and transferred onto a polyvinylidene difluoride membrane (Bio-Rad Laboratories, Inc., Hercules, CA, USA). The membranes were blocked and subsequently hybridized with specific primary antibodies, followed by incubation with horseradish peroxidase-conjugated goat anti-rabbit IgG secondary antibody (cat. no. 21234; dilution, 1:5,000) or goat anti-mouse IgG secondary antibody (cat. no. 31430; dilution, 1:5,000) (Invitrogen; Thermo Fisher Scientific, Inc.).

Proteins were visualized using Western Blotting Plus Chemiluminescence reagents (Thermo Fisher Scientific, Inc.) and autoradiography. The density of each protein band was determined following normalization with the GAPDH control band by using a gel image system and ImageJ software (version 2.0; National Institutes of Health).

Mouse xenografts. Animal procedures were ethically approved by the Institutional Animal Care and Use Committee of Chang Gung University (Taoyuan, Taiwan) and conformed to the guidelines of the research council for the care and use of laboratory animals at our institution. All animals were allowed free access to chlorinated water and irradiated food. The mice were housed in individually ventilated cages with a ventilation rate of 65 exchanges of fresh air per hour, a controlled temperature of $22^{\circ} \mathrm{C}$ and a $12 / 12$-h light-dark cycle. The human end points for tumor model was approved by IACUC in this study included: Tumor volume reaching 2,500 $\mathrm{mm}^{3}$ and more than $10 \%$ body weight loss. A total of 5 five-week-old male BALB/cAnN.Cg-Foxn1 ${ }^{\text {nu}} /$ CrlNarl mice (18-22 g; National Laboratory Animal Center, Taipei, Taiwan) were used, and $10^{6} \mathrm{SAS}$ cells were subcutaneously injected into the upper portion of the hind limb. After 7 days, PL $(2.4 \mathrm{mg} / \mathrm{kg})$ was intraperitoneally administered daily for 13 consecutive days. The tumor size was monitored, and the volume was calculated using the following equation: Tumor volume: (Short length of the tumor ${ }^{2} \mathrm{x}$ long length of the tumor)/2. Mice were sacrificed 6 weeks after the first PL administration, and the xenograft tumors were removed and weighed. Xenografted tumors were fixed in $3.7 \%$ formaldehyde solution for $24 \mathrm{~h}$ at room temperature and embedded in paraffin. Each sample was counterstained with a hematoxylin and eosin stain (H\&E) according to the manufacturer's protocol (Zymed; Thermo Fisher Scientific, Inc.).

Statistical analyses. Student's unpaired t-test was used to compare the difference between two groups. Statistical comparisons between multiple groups were performed using 
A
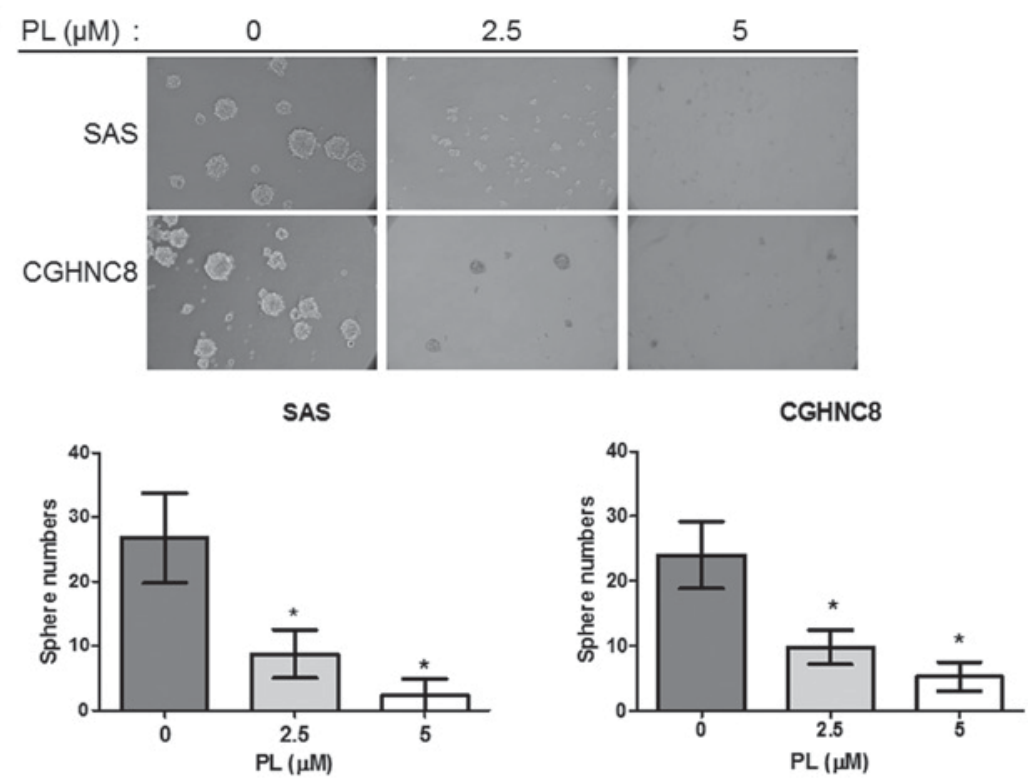

B
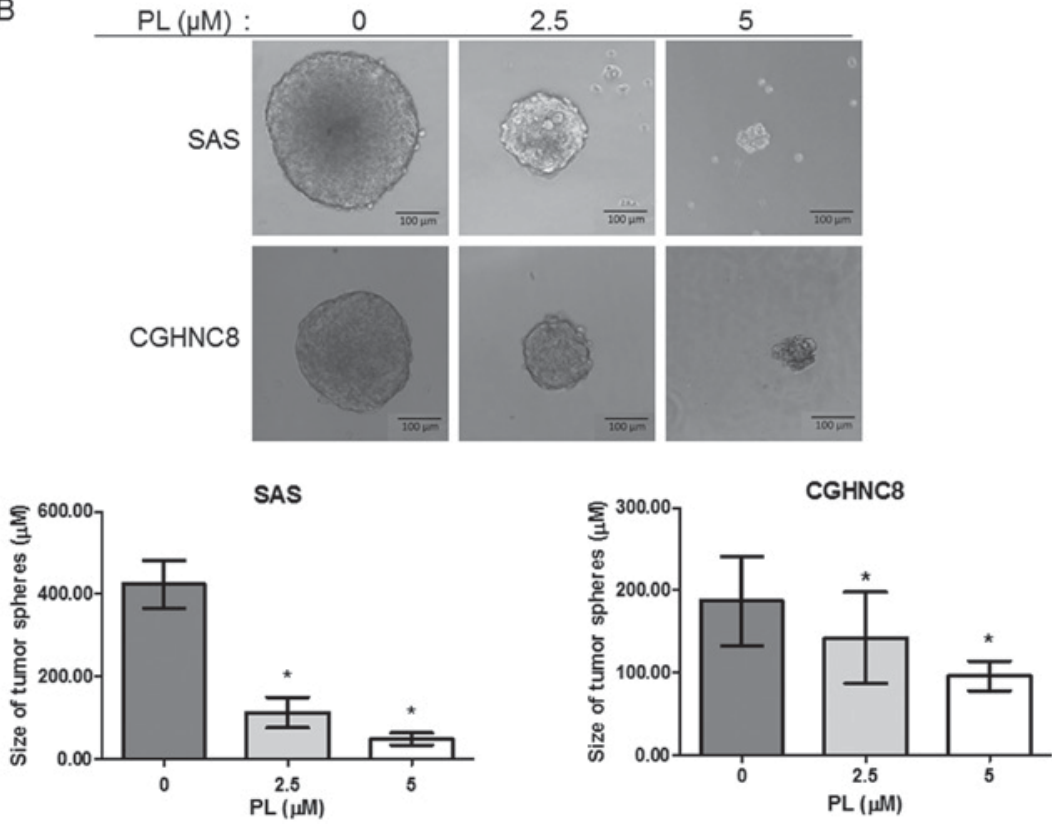

Figure 1. PL suppressed cancer stem cell properties including tumor sphere-forming ability. (A) Representative image of the number (magnification, $\mathrm{x} 40$ ) and (B) size of tumor spheres (magnification, $\mathrm{x} 400$ ) formed by SAS and CGHNC8 cells following treatment with 0.0 (0.1\% DMSO), 2.5 and 5.0 $\mu \mathrm{M}$ PL. The bar graphs present the number and diameter of the tumorspheres (scale bar, $100 \mu \mathrm{m}$ ). ${ }^{*} \mathrm{P}<0.05$ vs. the untreated control group. PL, piperlongumine.

a one-way analysis of variance and Tukey's post hoc test. The results were presented as the mean \pm the standard deviation (SD). All of the figures were plotted using GraphPad Prism (version 6.0; GraphPad Software Inc., La Jolla, CA, USA). $\mathrm{P}<0.05$ was considered to indicate a statistically significant difference.

\section{Results}

PL inhibits CSC properties. In the present study, a tumorsphere formation assay was used to investigate the function of $\mathrm{PL}$ in regulating CSC properties following treatment of SAS and CGHNC8 cells with PL $(0.0,2.5$ and 5.0 $\mu \mathrm{M})$. PL inhibited the number and size of tumor spheres formed by the tested cell lines in a concentration-dependent manner.
Following treatment with 2.5 and $5.0 \mu \mathrm{M}$ PL, the number of tumorspheres was reduced to $\sim 67.28$ and $91.58 \%$, respectively, compared with control cells, and similar effects were observed in the CGHNC8 cells (Fig. 1A). The average diameters of the tumorspheres were 418.33 and $190.88 \mu \mathrm{m}$ in the control SAS and CGHNC8 cells, respectively. By contrast, treatment with $5 \mu \mathrm{M}$ PL induced the formation of small spheres with diameters of 48.27 and $95.62 \mu \mathrm{m}$ in the SAS and CGHNC8 cells, respectively (Fig. 1B). Furthermore, the present study examined the alteration of the expression of CSC-associated genes and proteins following PL treatment. Transcription factors including Oct-4, NANOG and SOX2 regulate the pluripotency of embryonic stem cells, and CK18 participates in cell differentiation $(34,35)$. Following PL treatment, the mRNA levels of SOX2, NANOG, and Oct-4 decreased to 
A
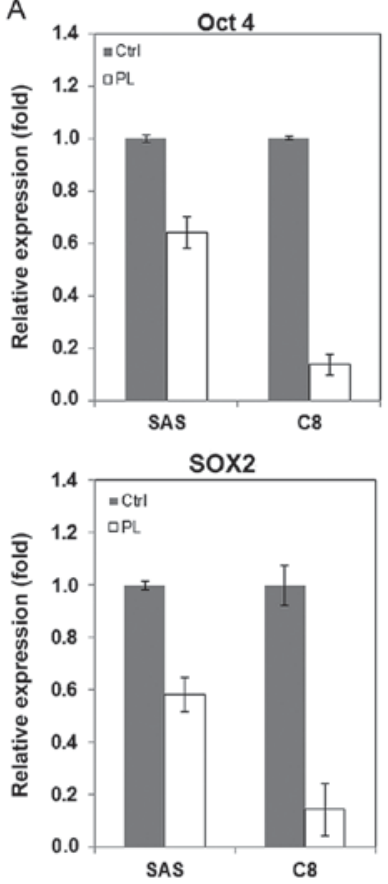
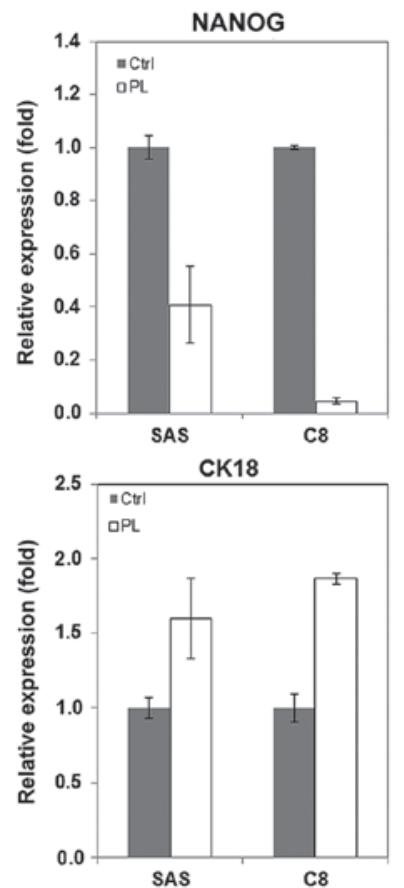

B

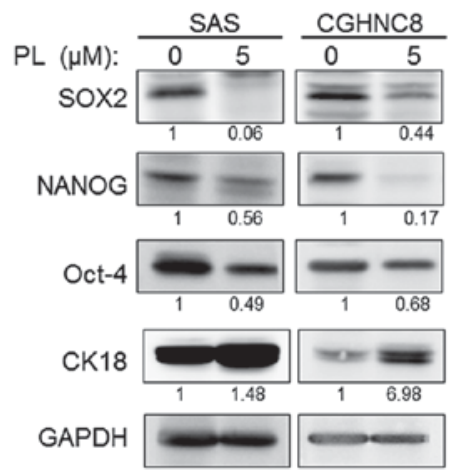

Figure 2. PL reduced the expression of the cancer stem cell markers SOX2, Oct-4, and NANOG but increased the expression of the differentiation marker CK18. SAS and CGHNC8 cells were treated with $5.0 \mu \mathrm{M}$ PL for $48 \mathrm{~h}$; subsequently, the cells were harvested and analyzed. (A) mRNA expression levels of SOX2, NANOG, and Oct-4 and CK18 were measured using reverse transcription-quantitative polymerase chain reaction. (B) Cellular protein levels were determined using western blotting (control cells, $0.1 \%$ DMSO). Experiments were performed in triplicate. PL, piperlongumine; SOX2, SRY-box 2; Oct-4, POU class 5 homeobox 1; NANOG, NANOG homeobox; CK18, cytokeratin 18 .

42,60 and $36 \%$ respectively in the SAS cells and to 86,96 and $87 \%$ respectively in the CGHNC8 cells, compared with control cells (Fig. 2A). Furthermore, following PL treatment, the CK18 expression levels increased 1.6 and 1.8 times in the SAS and CGHNC8 cells, respectively. Similarly, PL reduced Oct-4, NANOG, and SOX2 protein levels and increased CK18 protein levels (Fig. 2B). These results suggest that PL inhibits tumor sphere-forming ability via the suppression of Oct-4, NANOG, and SOX2 expression and promoting cell differentiation.

$P L$ reduces cell migration and invasion ability and EMT. As CSCs are invasive, the present study examined the potential effect of PL on cell invasion. Cell migration and invasion were determined using wound healing and Matrigel Transwell assays, respectively. Although different cell lines migrated at different rates, the control cells covered the majority of the wound area; in comparison, fewer PL-treated cells migrated to the wound area (8\% in the SAS cells and $61 \%$ in the CGHNC8 cells) after $10 \mathrm{~h}$ (Fig. 3A). The Matrigel invasion assay revealed that after $18 \mathrm{~h}$ of PL treatment, the number of invasive cells for the SAS and CGHNC8 cell lines decreased compared with that observed for the control cells (Fig. 3B). PL reduced the cell migration and invasion ability of the two cell lines in a concentration-dependent manner. EMT is another biological process that enables the metastasis of tumor cells through the bloodstream. Similar to the cancer stemness pathway, this process mechanistically initiates cancer metastasis and confers treatment resistance (36). Therefore, the function that PL serves in EMT was investigated. Compared with the control cells, the PL-treated cells exhibited an increased expression level of E-cadherin but a decreased expression level of N-cadherin and vimentin. PL reduced the levels of transcription factors, including Snail and Slug that participate in EMT (Fig. 3C). These results suggest that PL suppressed cell migration and invasion ability, and so regulated EMT in oral cancer cells.

PL increases radio- and chemosensitivity. Surgery, radiotherapy, chemotherapy or combinations of these methods are the main therapeutic methods for oral cancer. The main reason for therapeutic failure is acquired resistance, which is attributed to CSCs. Therefore, whether PL serves as a sensitizer was investigated in order to improve therapeutic efficacy. The clonogenic survival assay was used to examine the radiosensitization effect of PL following the treatment of cells with PL alone, radiation alone or a combination of PL and radiation. The radiation sensitivity levels of SAS and CGHNC8 cells treated with the combination of PL and radiation increased by 47.5 and $25.63 \%$, respectively (Fig. $4 \mathrm{~A}$ ). 5 -FU, which is commonly used for treating oral cavity cancers, was selected to test chemosensitivity. As presented in Fig. 4B, 60 and 70\% of SAS and CGHNC8 cells, respectively, were killed following treatment with 5-FU for $48 \mathrm{~h}$. The same effect was observed in PL-treated cells. The SAS and CGHNC8 cells that were treated with 5-FU and PL exhibited enhanced drug sensitivity (Fig. 4B). Thus, PL enhanced the radio- and chemosensitivity levels of oral cancer cells.

PL inhibits tumor growth ability in vitro and in vivo. PL induces apoptosis in various types of cancer $(19,20)$. Therefore, whether PL regulates the tumor growth ability of oral cancer cells was investigated. PL treatment $(5 \mu \mathrm{M})$ for $48 \mathrm{~h}$ reduced the growth of the SAS and CGHNC8 cells to 11.2 and 21.9\%, respectively, compared with the control cells (Fig. 5A). The colony formation assay was used to examine the long-term effect of PL by treating cells with PL $(0.625$ and $2.500 \mu \mathrm{M})$ for 7 days. Consistently, PL significantly suppressed colony formation, and no or few colonies were formed following PL treatment $(2.5 \mu \mathrm{M}$; Fig. 5B). Furthermore, PL reduced the growth of oral cancer cells and their colony-forming ability. In order to investigate the effects of PL on tumor growth in vivo, xenograft SAS tumors were established in BALB/c nude mice. Following injection with SAS tumor cells, the mice (5 per treatment group) were intraperitoneally administered with 
A
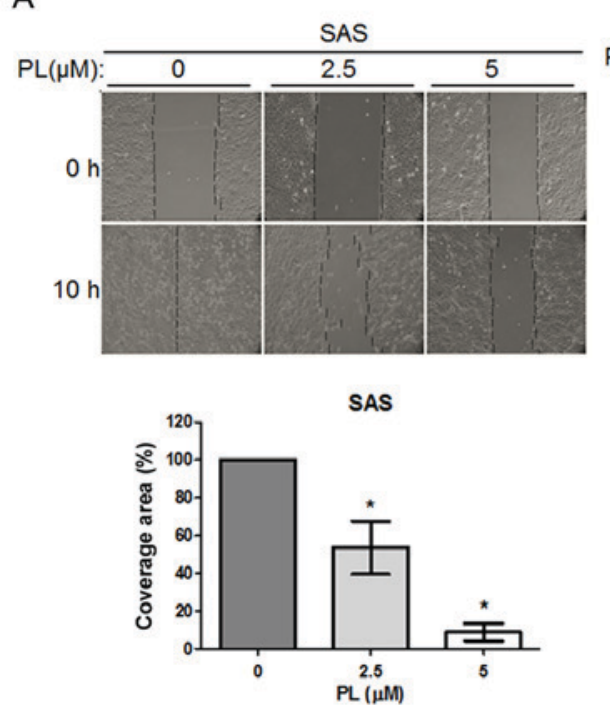

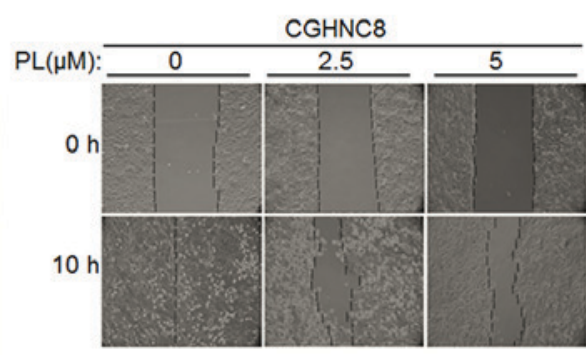

CGHNC8

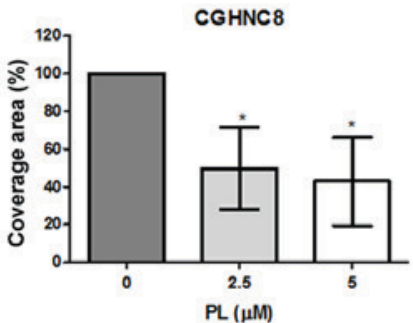

C

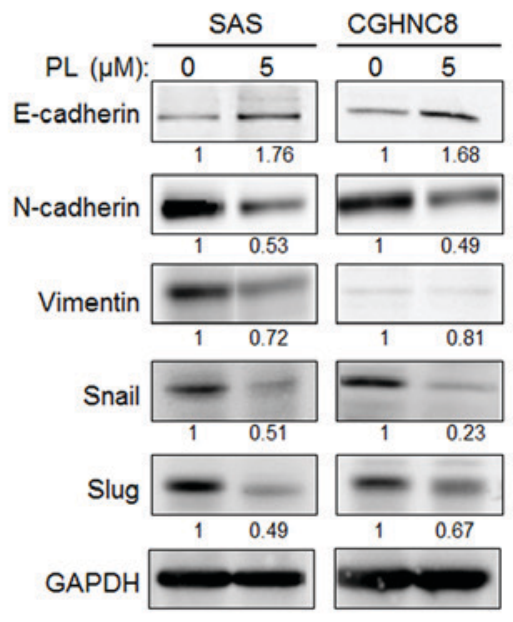

B
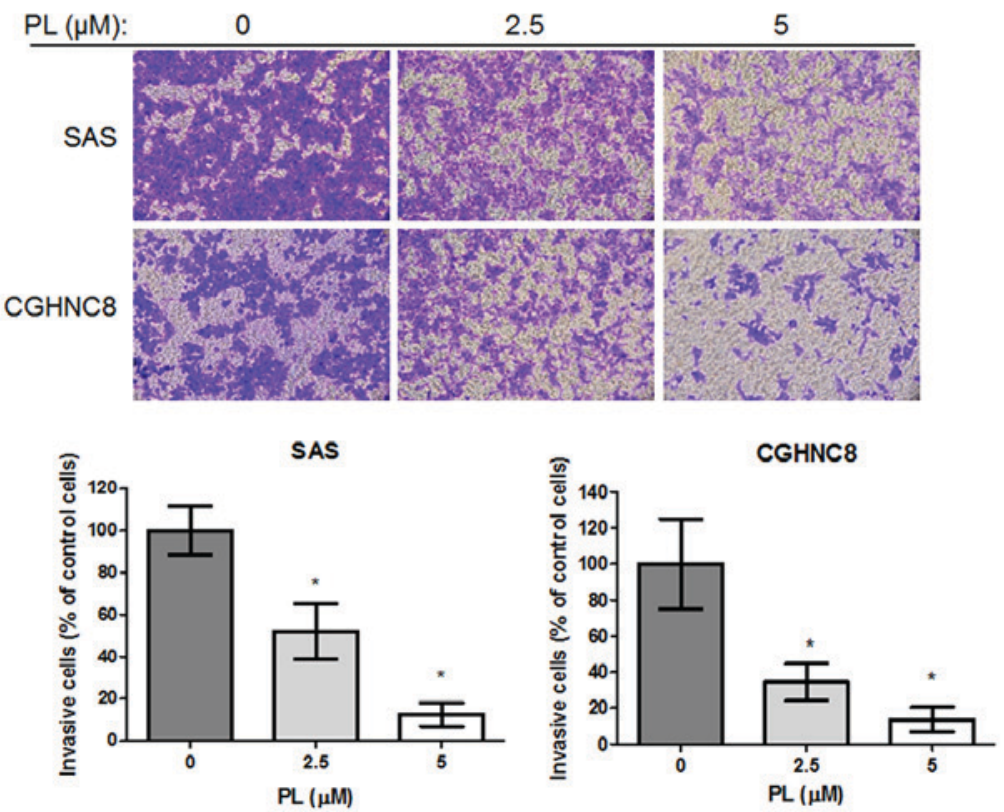

Figure 3. PL suppressed cell migration and invasion, and regulated the expression of EMT-associated markers. (A) PL suppressed cell migration, revealed using a wound healing assay. (B) PL suppressed cell invasion ability, revealed using a Matrigel invasion assay. (C) Western blotting was used to examine the expression of EMT-associated molecules. "P<0.05 vs. the untreated control group. PL, piperlongumine; EMT, epithelial-mesenchymal transition; E-, epithelial; N-, neural; Snail1, Snail family transcriptional repressor 1; Slug, Snail family transcriptional repressor 2.

PL or control saline and monitored for 6 consecutive weeks. Tumor growth was significantly slower in the PL-treated groups than in the control groups (Fig. 5C). On day 42, tumor growth decreased by $63 \%$ in the PL-treated group $(\mathrm{P}<0.05)$. Once the mice were sacrificed, the tumor weight was revealed to be decreased by $66 \%$ in the PL-treated group (Fig. 5D). Tumor tissues were confirmed using H\&E staining (Fig. 5E). The results indicate that PL inhibited tumor growth in vitro and in vivo.

\section{Discussion}

CSCs serve a pivotal function not only in the therapy resistance phenotype but also in local recurrence and metastasis (8). CSCs may generate tumors through the stem cell processes of self-renewal and differentiation (4,37). Therefore, eliminating
CSCs may provide a therapeutic strategy for cancer. In the present study, PL was demonstrated to suppress the tumor sphere-forming ability of the oral cancer cells SAS and CGHNC8 (Fig. 1) and suppress the expression of the stem cell-associated genes SOX2, Oct-4, and NANOG. It was demonstrated to increase the expression of the differentiation gene CK18 (Fig. 2). Furthermore, PL inhibited cell migration and invasion and regulated the expression of EMT-associated molecules (Fig. 3). PL also increased chemo- and radiosensitivity (Fig. 4) and inhibited tumorigenesis in vitro and in vivo (Fig. 5). Oct-4, NANOG, and SOX2 are essential for early embryonic development and pluripotency maintenance in embryonic stem cells $(38,39)$. These factors regulate the cancer stemness phenotype and are upregulated in multiple types of cancer. They thus serve a critical function in cancer development and drug resistance $(34,40,41)$. PL has been 
A

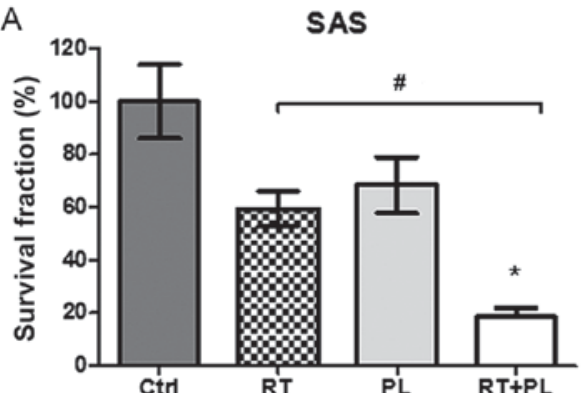

B

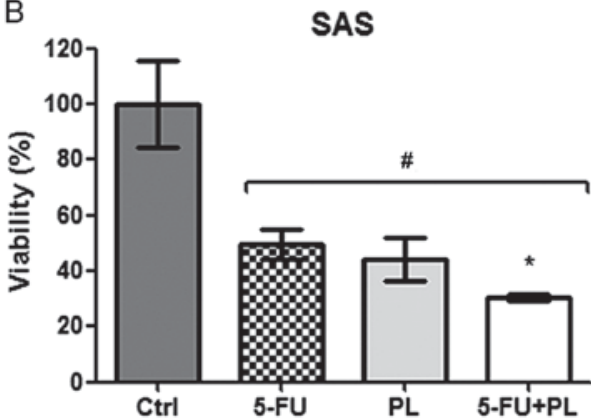

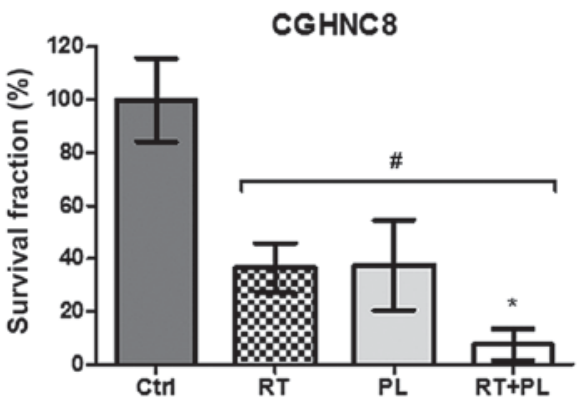

CGHNC8

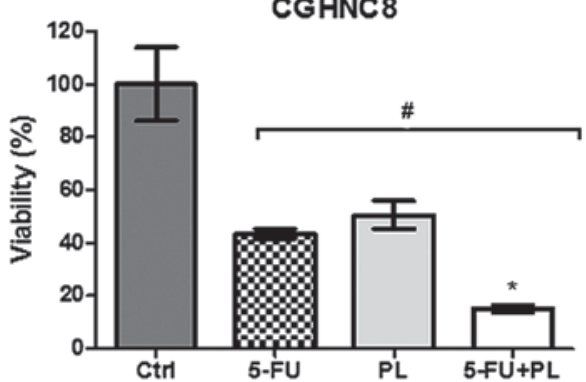

Figure 4. PL increased the radio- and chemosensitivity levels of oral cancer cells. (A) Clonogenic survival assays were used to determine the effect of PL on the radiosensitivity of SAS and CGHNC8 cells. Cells were treated with either PL alone $(0.625 \mu \mathrm{M})$, radiation alone (2 Gy) or a combination of the two treatments (B) PL increased chemosensitivity, as determined by the cytotoxic assay. Cells were treated with $0.03 \mu \mathrm{M}$ 5-FU alone, $2.50 \mu \mathrm{M}$ PL alone, or a combination of the two treatments for $48 \mathrm{~h}$. Cell survival was examined using an MTS assay. The data of the cytotoxic assay were compared with the data of control cells Experiments were performed in triplicate. " $\mathrm{P}<0.05$ vs. the ctrl or RT/5-FU treated groups, ${ }^{\text {"P }}<0.05$ vs. the RT/5-FU or RT+PL/5-FU+PL treated groups, with comparisons indicated by lines. PL, piperlongumine; Ctrl, control; RT, radiotherapy; 5-FU, 5-fluorouracil.

identified as an inhibitor of signal transducer and activator of transcription 3 (STAT3) from the screening of a chemical library (22). PL inhibits STAT3 nuclear translocation, suppresses ligand-induced and constitutive STAT3 phosphorylation and affects STAT3-regulated gene expression in breast cancer cells (22). STAT3 is crucial for embryonic stem cell pluripotency and is essential for the maintenance of inner cell mass lineages through the downstream effectors Oct- 4 and NANOG $(42,43)$. Therefore, PL may suppress cancer stemness by inhibiting STAT3. The results of the present study demonstrate that PL inhibited CSC formation and SOX2, Oct-4 and NANOG expression, supporting the observation that PL suppresses CSC properties in oral cancer.

A direct link exists between EMT and CSCs. For example, EMT may induce a more CSC-like phenotype in various types of cancer $(8,9,44)$. Furthermore, CSC markers are associated with tumorigenesis, metastasis and recurrence. For example, oct-4 is highly expressed in invasive oral cancer cells, and this expression is accompanied by alterations in the expression of EMT-associated markers, low E-cadherin expression and high $\mathrm{N}$-cadherin expression (44). The coexpression of Oct-4 and NANOG enhances CSC-like properties, modulates STAT3 activation and subsequently regulates Snail expression and promotes cell metastatic ability in hepatocellular carcinoma (45). Snail and Slug are the major transcription factors that repress epithelial marker genes and activate mesenchymal markers to promote cell mobility. Snail- and Slug-induced EMT triggers CSC-like phenotypes in multiple types of cancer (46-49). In the present study, PL was demonstrated to reduce cancer stemness by inhibiting tumor sphere formation and suppressing SOX2, Oct-4 and NANOG expression; however, PL increased the expression of the differentiation marker CK18 (Figs. 1 and 2). Furthermore, PL inhibited cell mobility, which was accompanied by the regulation of the expression of EMT-associated molecules. PL-treated cells exhibited a high expression of E-cadherin and a low expression of mesenchymal markers including $\mathrm{N}$-cadherin and vimentin. In addition, PL reduced the levels of transcription factors Snail and Slug (Fig. 3). PL was demonstrated to suppress cancer stemness, eliminate EMT processes and suppress the differential expression of EMT-associated molecules.

For oral cancer therapy, radiotherapy alone may be used to treat small or early-stage tumors. Radiotherapy is commonly used following surgery, either alone or in combination with chemotherapy, in order to treat advanced tumors. ROS are critical mediators of ionization-induced cell death, and lower ROS levels have been observed in CSCs exhibiting greater resistance to radiotherapy (50). Therefore, modulating ROS levels may be a useful method to improve the efficacy of radiotherapy. A number of studies have indicated that PL increases ROS generation in cancer cells, but not in normal cells, and enhanced ROS levels are associated with cancer cell death $(19,23,51)$. Thus, the increased ROS production by PL suggests that PL may serve as a radiosensitizer in order to improve the therapeutic efficacy of radiotherapy. In the present study, it was demonstrated that pretreatment with PL increased the antitumor effect of radiotherapy (Fig. 4). Similar results were observed in a breast cancer model. The cell apoptosis rate increased following combined treatment of PL with radiation, and the level of intracellular ROS was higher in the combination group than in the group treated with $\mathrm{PL}$ or radiation alone (17). These results may be attributed to the decreased expression level of BCL2 and the increased expression level of BCL2 associated $\mathrm{X}$, apoptosis regulator. 
A

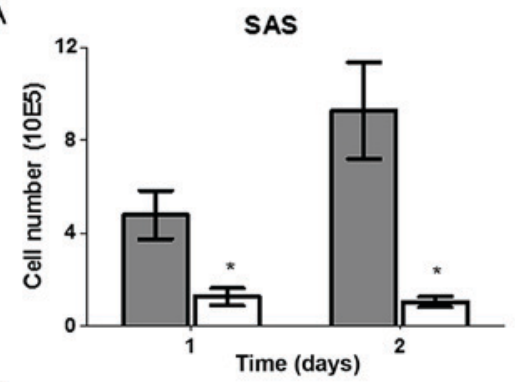

B

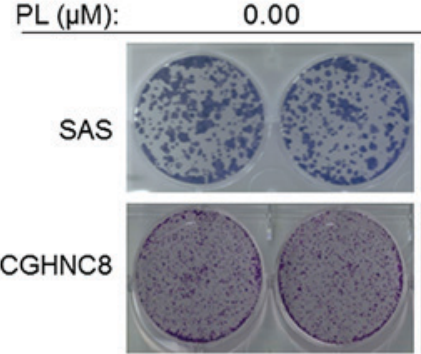

C

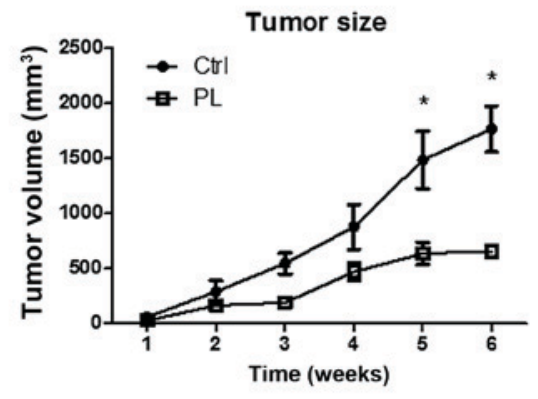

E

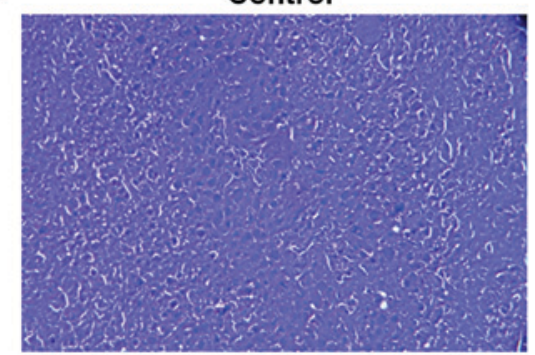

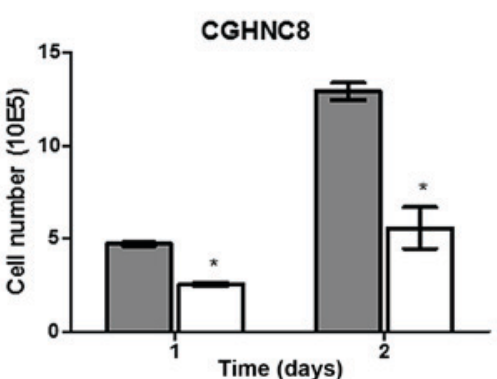

0.625

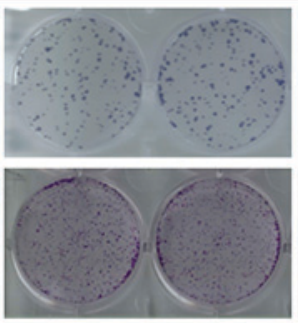

D

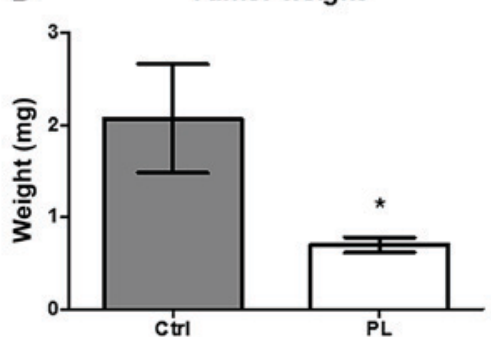

PL

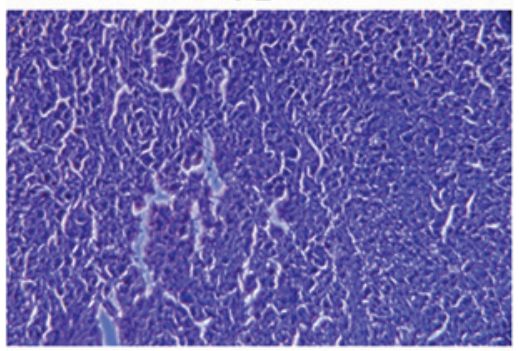

Figure 5. PL suppressed cell growth. (A) PL suppressed tumor growth in vitro, as determined by the cell number enumeration. (B) PL suppressed colony formation, demonstrated using a colony formation assay. (C) PL reduced tumor growth in xenografted tumors. SAS cells were subcutaneously injected into BALB/c nude mice. After 7 days, PL was injected intraperitoneally. The tumor size was measured weekly following injection. (D) Once the mice were sacrificed, the tumors were removed and weighed. (E) The grafted tumors were confirmed by hematoxylin and eosin staining. The results are presented as the mean \pm the standard deviation. ${ }^{*} \mathrm{P}<0.05$ vs. the untreated control group. PL, piperlongumine; Ctrl, control.

Drug resistance may be acquired due to the presence of CSCs and elevated CSC markers (3,4,40). PL has been demonstrated to increase 5-FU sensitivity in oral cancer (Fig. 4). PL exhibits a synergistic effect with various chemotherapeutic drugs, including cisplatin and paclitaxel, in ovarian, head and neck cancer $(24,26,52)$. PL suppressed the expression of P-glycoprotein, ATP binding cassette subfamily B member 1, ATP binding cassette subfamily $\mathrm{C}$ member 1 , survivin, and phospho-Akt, as well as the transcriptional activities of NF- $\mathrm{KB}$ and TWIST, which reversed doxorubicin resistance in a breast cancer cell line (53).

In conclusion, PL increases therapeutic sensitivity and suppresses the malignant phenotype, namely via the suppression of migration, invasion, EMT and proliferation in vitro and in vivo by inhibiting cancer stemness and the expression of stem cell regulatory proteins. This therefore indicates that PL is a potential therapeutic agent for oral cancer.

\section{Acknowledgements}

The present study was supported by the Taipei Medical University Hospital (grant no. 102TMU-TMUH-06) and the Ministry of Science and Technology, Taiwan, R.O.C (grant no. MOST 104-2320-B-038-032).

\section{References}

1. Prince ME, Sivanandan R, Kaczorowski A, Wolf GT, Kaplan MJ, Dalerba P, Weissman IL, Clarke MF and Ailles LE: Identification of a subpopulation of cells with cancer stem cell properties in head and neck squamous cell carcinoma. Proc Natl Acad Sci USA 104: 973-978, 2007.

2. Visvader JE and Lindeman GJ: Cancer stem cells in solid tumours: Accumulating evidence and unresolved questions. Nat Rev Cancer 8: 755-768, 2008

3. Rich JN: Cancer stem cells in radiation resistance. Cancer Res 67: 8980-8984, 2007. 
4. Khan IN, Al-Karim S, Bora RS, Chaudhary AG and Saini KS: Cancer stem cells: A challenging paradigm for designing targeted drug therapies. Drug Discov Today 20: 1205-1216, 2015.

5. Gupta GP and Massagué J: Cancer metastasis: Building a framework. Cell 127: 679-695, 2006.

6. Thiery JP and Sleeman JP: Complex networks orchestrate epithelial-mesenchymal transitions. Nat Rev Mol Cell Biol 7: 131-142, 2006

7. Thiery JP, Acloque H, Huang RY and Nieto MA: Epithelialmesenchymal transitions in development and disease. Cell 139 871-890, 2009

8. Sampieri K and Fodde R: Cancer stem cells and metastasis. Semin Cancer Biol 22: 187-193, 2012.

9. Scheel C and Weinberg RA: Cancer stem cells and epithelial-mesenchymal transition: Concepts and molecular links. Semin Cancer Biol 22: 396-403, 2012.

10. Barbazán J, Muinelo-Romay L, Vieito M, Candamio S, Díaz-López A, Cano A, Gómez-Tato A, Casares de Cal Mde L, Abal M and López-López R: A multimarker panel for circulating tumor cells detection predicts patient outcome and therapy response in metastatic colorectal cancer. Int J Cancer 135: 2633-2643, 2014

11. Warnakulasuriya S: Global epidemiology of oral and oropharyngeal cancer. Oral Oncol 45: 309-316, 2009.

12. Huang SH and O'Sullivan B: Oral cancer: Current role of radiotherapy and chemotherapy. Med Oral Patol Oral Cir Bucal 18 e233-e240, 2013

13. Chang JT, Chen IH, Liao CT, Wang HM, Hsu YM, Hung KF, Lin CJ, Hsieh LL and Cheng AJ: A reverse transcription comparative real-time PCR method for quantitative detection of angiogenic growth factors in head and neck cancer patients. Clin Biochem 35: 591-596, 2002.

14. Chen YJ, Chang JT, Liao CT, Wang HM, Yen TC, Chiu CC $\mathrm{Lu} \mathrm{YC}, \mathrm{Li} \mathrm{HF}$ and Cheng AJ: Head and neck cancer in the betel quid chewing area: Recent advances in molecular carcinogenesis. Cancer Sci 99: 1507-1514, 2008.

15. Liao CT, Chang JT, Wang HM, Ng SH, Hsueh C, Lee LY, Lin $\mathrm{CH}$, Chen IH, Huang SF, Cheng AJ and Yen TC: Analysis of risk factors of predictive local tumor control in oral cavity cancer. Ann Surg Oncol 15: 915-922, 2008.

16. Lee W, Yoo H, Kim JA, Lee S, Jee JG, Lee MY, Lee YM and Bae JS: Barrier protective effects of piperlonguminine in LPS-induced inflammation in vitro and in vivo. Food Chem Toxicol 58: 149-157, 2013

17. Yao L, Chen HP and Ma Q: Piperlongumine alleviates lupus nephritis in MRL-Fas(lpr) mice by regulating the frequency of Th17 and regulatory T cells. Immunol Lett 161: 76-80, 2014.

18. Son DJ, Kim SY, Han SS, Kim CW, Kumar S, Park BS, Lee SE, Yun YP, Jo H and Park YH: Piperlongumine inhibits atherosclerotic plaque formation and vascular smooth muscle cell proliferation by suppressing PDGF receptor signaling. Biochem Biophys Res Commun 427: 349-354, 2012.

19. Raj L, Ide T, Gurkar AU, Foley M, Schenone M, Li X, Tolliday NJ, Golub TR, Carr SA, Shamji AF, et al: Selective killing of cancer cells by a small molecule targeting the stress response to ROS. Nature 475: 231-234, 2011.

20. Bezerra DP, Pessoa C, de Moraes MO, Saker-Neto N, Silveira ER and Costa-Lotufo LV: Overview of the therapeutic potential of piplartine (piperlongumine). Eur J Pharm Sci 48: 453-463, 2013.

21. Randhawa H, Kibble K, Zeng H, Moyer MP and Reindl KM: Activation of ERK signaling and induction of colon cancer cell death by piperlongumine. Toxicol In Vitro 27: 1626-1633, 2013.

22. Bharadwaj U, Eckols TK, Kolosov M, Kasembeli MM, Adam A Torres D, Zhang X, Dobrolecki LE, Wei W, Lewis MT, et al: Drug-repositioning screening identified piperlongumine as a direct STAT3 inhibitor with potent activity against breast cancer. Oncogene 34: 1341-1353, 2015.

23. Jin HO, Lee YH, Park JA, Lee HN, Kim JH, Kim JY, Kim B, Hong SE, Kim HA, Kim EK, et al: Piperlongumine induces cell death through ROS-mediated CHOP activation and potentiates TRAIL-induced cell death in breast cancer cells. J Cancer Res Clin Oncol 140: 2039-2046, 2014.

24. Roh JL, Kim EH, Park JY, Kim JW, Kwon M and Lee BH: Piperlongumine selectively kills cancer cells and increases cisplatin antitumor activity in head and neck cancer. Oncotarget 5 9227-9238, 2014

25. Shrivastava S, Kulkarni P, Thummuri D, Jeengar MK, Naidu VG, Alvala M, Redddy GB and Ramakrishna S: Piperlongumine, an alkaloid causes inhibition of PI3K/Akt/mTOR signaling axis to induce caspase-dependent apoptosis in human triple-negative breast cancer cells. Apoptosis 19: 1148-1164, 2014.
26. Wang XQ, Wang YC, Guo YT and Tang X: Effect of piperlongumine on drug resistance reversal in human retinoblastoma HXO-RB44/VCR and SO-Rb50/CBP cell lines. Int J Clin Exp Pathol 8: 2525-2534, 2015.

27. Bleau AM, Hambardzumyan D, Ozawa T, Fomchenko EI, Huse JT, Brennan CW and Holland EC: PTEN/PI3K/Akt pathway regulates the side population phenotype and ABCG2 activity in glioma tumor stem-like cells. Cell Stem Cell 4: 226-235, 2009.

28. Shostak K and Chariot A: NF- $\kappa \mathrm{B}$, stem cells and breast cancer: The links get stronger. Breast Cancer Res 13: 214, 2011.

29. Matsuda K, Sato A, Okada M, Shibuya K, Seino S, Suzuki K, Watanabe E, Narita Y, Shibui S, Kayama T and Kitanaka C: Targeting JNK for therapeutic depletion of stem-like glioblastoma cells. Sci Rep 2: 516, 2012.

30. Lu YC, Chen YJ, Wang HM, Tsai CY, Chen WH, Huang YC, Fan KH, Tsai CN, Huang SF, Kang CJ, et al: Oncogenic function and early detection potential of miRNA-10b in oral cancer as identified by microRNA profiling. Cancer Prev Res (Phila) 5: 665-674, 2012

31. Chen CY, Lin YS, Chen CL, Chao PZ, Chiou JF, Kuo CC, Lee FP, Lin YF, Sung YH, Lin YT, et al: Targeting annexin A2 reduces tumorigenesis and therapeutic resistance of nasopharyngeal carcinoma. Oncotarget 29: 26946-26959, 2015.

32. Livak KJ and Schmittgen TD: Analysis of relative gene expression data using real-time quantitative PCR and the 2(-Delta Delta C(T)) method. Methods 25: 402-408, 2001.

33. Chen YJ, Lee LY, Chao YK, Chang JT, Lu YC, Li HF, Chiu CC, Li YC, Li YL, Chiou JF and Cheng AJ: DSG3 facilitates cancer cell growth and invasion through the DSG3-plakoglobin-TCF/LEFMyc/cyclin D1/MMP signaling pathway. PLoS One 8: e64088, 2013.

34. Guo Y, Liu S, Wang P, Zhao S, Wang F, Bing L, Zhang Y, Ling EA, Gao J and Hao A: Expression profile of embryonic stem cell-associated genes Oct4, Sox 2 and Nanog in human gliomas. Histopathology 59: 763-775, 2011.

35. Chiu CC, Lee LY, Li YC, Chen YJ, Lu YC, Li YL, Wang HM, Chang JT and Cheng AJ: Grp78 as a therapeutic target for refractory head-neck cancer with CD24(-)CD44(+) stemness phenotype. Cancer Gene Ther 20: 606-615, 2013.

36. Brabletz T: EMT and MET in metastasis: Where are the cancer stem cells? Cancer Cell 22: 699-701, 2012.

37. Clarke MF, Dick JE, Dirks PB, Eaves CJ, Jamieson CH, Jones DL, Visvader J, Weissman IL and Wahl GM: Cancer stem cells-perspectives on current status and future directions: AACR Workshop on cancer stem cells. Cancer Res 66: 9339-9344, 2006

38. Boyer LA, Lee TI, Cole MF, Johnstone SE, Levine SS, Zucker JP, Guenther MG, Kumar RM, Murray HL, Jenner RG, et al: Core transcriptional regulatory circuitry in human embryonic stem cells. Cell 122: 947-956, 2005.

39. Loh YH, Wu Q, Chew JL, Vega VB, Zhang W, Chen X, Bourque G, George J, Leong B, Liu J, et al: The Oct4 and Nanog transcription network regulates pluripotency in mouse embryonic stem cells. Nat Genet 38: 431-440, 2006.

40. Tsai LL, Yu CC, Chang YC, Yu CH and Chou MY: Markedly increased Oct4 and Nanog expression correlates with cisplatin resistance in oral squamous cell carcinoma. J Oral Pathol Med 40: 621-628, 2011.

41. Ling GQ, Chen DB, Wang BQ and Zhang LS: Expression of the pluripotency markers Oct $3 / 4$, Nanog and Sox 2 in human breast cancer cell lines. Oncol Lett 4: 1264-1268, 2012

42. Niwa H, Burdon T, Chambers I and Smith A: Self-renewal of pluripotent embryonic stem cells is mediated via activation of STAT3. Genes Dev 12: 2048-2060, 1998.

43. Do DV, Ueda J, Messerschmidt DM, Lorthongpanich C, Zhou Y, Feng B, Guo G, Lin PJ, Hossain MZ, Zhang W, et al: A genetic and developmental pathway from STAT3 to the OCT4-NANOG circuit is essential for maintenance of ICM lineages in vivo. Genes Dev 27: 1378-1390, 2013.

44. Siu A, Lee C, Dang D and Ramos DM: Stem cell markers as predictors of oral cancer invasion. Anticancer Res 32: 1163-1166, 2012.

45. Yin X, Zhang BH, Zheng SS, Gao DM, Qiu SJ, Wu WZ and Ren ZG: Coexpression of gene Oct4 and Nanog initiates stem cell characteristics in hepatocellular carcinoma and promotes epithelial-mesenchymal transition through activation of Stat3/Snail signaling. J Hematol Oncol 8: 23, 2015.

46. Fan F, Samuel S, Evans KW, Lu J, Xia L, Zhou Y, Sceusi E, Tozzi F, Ye XC, Mani SA and Ellis LM: Overexpression of snail induces epithelial-mesenchymal transition and a cancer stem cell-like phenotype in human colorectal cancer cells. Cancer Med 1: 5-16, 2012. 
47. Guo W, Keckesova Z, Donaher JL, Shibue T, Tischler V, Reinhardt F, Itzkovitz S, Noske A, Zürrer-Härdi U, Bell G, et al: Slug and Sox 9 cooperatively determine the mammary stem cell state. Cell 148: 1015-1028, 2012.

48. Yasui K, Shimamura M, Mitsutake N and Nagayama Y: SNAIL induces epithelial-to-mesenchymal transition and cancer stem cell-like properties in aldehyde dehydroghenase-negative thyroid cancer cells. Thyroid 23: 989-996, 2013.

49. Masui T, Ota I, Yook JI, Mikami S, Yane K, Yamanaka T and Hosoi H: Snail-induced epithelial-mesenchymal transition promotes cancer stem cell-like phenotype in head and neck cancer cells. Int J Oncol 44: 693-699, 2014.

50. Diehn M, Cho RW, Lobo NA, Kalisky T, Dorie MJ, Kulp AN, Qian D, Lam JS, Ailles LE, Wong M, et al: Association of reactive oxygen species levels and radioresistance in cancer stem cells. Nature 458: 780-783, 2009.
51. Yao JX, Yao ZF, Li ZF and Liu YB: Radio-sensitization by Piper longumine of human breast adenoma MDA-MB-231 cells in vitro. Asian Pac J Cancer Prev 15: 3211-3217, 2014.

52. Han JG, Gupta SC, Prasad S and Aggarwal BB: Piperlongumine chemosensitizes tumor cells through interaction with cysteine 179 of IkappaBalpha kinase, leading to suppression of NF-кB-regulated gene products. Mol Cancer Ther 13: 2422-2435, 2014.

53. Kang Q and Yan S: Piperlongumine reverses doxorubicin resistance through the PI3K/Akt signaling pathway in K562/A02 human leukemia cells. Exp Ther Med 9: 1345-1350, 2015. 